\title{
MESOZOOPLÂNCTON DE ÁREA RECIFAL DO ATLÂNTICO SUDOESTE TROPICAL
}

\author{
Dilma Aguiar do NASCIMENTO-VIEIRA ${ }^{1,2}$ \\ Sigrid NEUMANN-LEITÃO ${ }^{1}$ \\ Fernando de Figueiredo PORTO NETO ${ }^{1,3}$ \\ Tâmara de Almeida e SILVA ${ }^{4}$ \\ Andréa Pinto SILVA ${ }^{1}$
}

Recebido: 01/04/10

Aceite: 01/06/10

\section{RESUMO}

Estudos sobre o zooplâncton foram realizados em uma área recifal do Atlântico Sudoeste Tropical (Tamandaré, Pernambuco, Brasil) visando conhecer as variações na biodiversidade e distribuição espacial e sazonal. Três estações foram amostradas no período de junho/89 a maio/90, nas preamares e baixa-mares diurnas. As amostras foram obtidas através de arrastos horizontais à superfície com rede de plâncton com 300 $\mu \mathrm{m}$ de abertura de malha. Dados de temperatura e salinidade foram coletados simultaneamente para fins comparativos. Foram identificados 47 taxa e o holoplâncton predominou com $61 \%$. Copepoda foi o grupo mais representativo, destacando-se Acartia (Odontacartia) lilljeborgi Giesbrecht, 1889 e Parvocalanus crassirostris (F. Dahl, 1894). Foi observado um ciclo sazonal com maiores densidades nos meses de verão nas três estações (máximo de 992 org. $\mathrm{m}^{-3}$ ), contudo os valores médios foram baixos $\left(295,7 \pm 259,4 \mathrm{org} \cdot \mathrm{m}^{-3}\right)$. A diversidade de espécies variou entre 2 e 3 bits.ind ${ }^{-1}$, baixa para área recifal. A presença de grande quantidade de espécies estuarinas evidenciou a conectividade entre estuários e a área recifal estudada.

Palavras chave: Biodiversidade, Copepoda, pluma estuarina, recifes costeiros, zooplâncton

\section{ABSTRACT}

Zooplankton studies were carried out in a reef area from the South Western Tropical Atlantic (Tamandaré, Pernambuco, Brazil) to assess the biodiversity and spatial and seasonal distribution. Three stations were sampled from June/89 to May/90 at low and high tides. Plankton samples were obtained by horizontal surface hauls with a plankton net with $300 \mu \mathrm{m}$ mesh size. Co-current temperature and salinity data were measured. It was identified 47 zooplanktonic taxa and the holoplankton dominated with $61 \%$. Copepoda was the most diverse and abundant, outranking Acartia (Odontacartia) lilljeborgi Giesbrecht, 1889 and Parvocalanus crassirostris (F. Dahl, 1894). A quantitative pattern was observed with higher densities at summer months (maximum 992 ind. $\mathrm{m}^{-3}$ ), however the average densities were low $\left(295.7 \pm 259.4\right.$ ind. $\left.\mathrm{m}^{-3}\right)$. Species diversity scored from 2 to 3 bits.ind $^{-1}$, low to a reef area. The presence of a high number of estuarine species show connectivity between estuaries and the reef area.

Key words: Biodiversity, Copepoda, estuarine plume, coastal reefs, zooplankton

\section{INTRODUÇÃO}

As regiões tropicais do mundo apresentam como regra geral uma baixa produtividade, em decorrência da termoclina permanente. Entretanto, existem três ambientes onde excepcionalmente a produtividade é alta: regiões de manguezais, de

Contatos: 1.Departamento de Oceanografia da UFPE, Av. Arquitetura, s/n, Cidade Universitária, 50.670-901, Recife, PE

2.Faculdade Salesiana do Nordeste, R. Dom Bosco, 551, Boa Vista, 50070-070, Recife, PE

3.Departamento de Zootecnia da UFRPE, R. Dom Manoel de Medeiros, s/n, Dois Irmãos, 52171-900

- Recife, PE

4.Universidade do Estado da Bahia, Departamento de Educação, Campus VIII 
NASCIMENTO-VIEIRA et al. Mesozooplâncton de área recifal do Atlântico Sudoeste Tropical

ressurgência e recifes de corais (GROSS; GROSS, 1996). Este último caracterizado também por alta biodiversidade.

Um dos maiores problemas relacionados à biodiversidade é que não são conhecidas as espécies de muitas áreas, tornando difícil avaliar o seu status, pois muitas espécies estão sendo eliminadas antes de serem conhecidas. Entre os ecossistemas mais citados pela falta de conhecimento de sua flora e fauna estão as áreas profundas do oceano e as áreas recifais (SOROKIN, 1990).

Uma das mais fortes razões para se manter a biodiversidade é a importância ecológica do ecossistema, isto é, a manutenção da sua função, pois a perda de espécies pode levar um ecossistema a não desempenhar mais o seu papel (NEW, 1995).

A costa nordestina do Brasil apresenta uma longa linha de recifes areníticos, sendo, particularmente os de Tamandaré do tipo "tacis" constituído, também, por corais hermatípicos, hidrocorais e algas calcáreas (MABESOONE; COUTINHO, 1970). Em alguns locais, onde o turismo é mais desenvolvido, estas áreas recifais passam a sofrer impactos diversos que levam ao desequilíbrio, favorecendo o desenvolvimento de espécies restrategista, com conseqüente queda na biodiversidade.

Nesses ecossistemas, o zooplâncton tem papel chave como elo na transferência da energia e da matéria, sendo uma das maiores fontes nutritivas para a fauna recifal (EREZ, 1990). Segundo Johannes (1975) muitos recifes de corais não poderiam subsistir unicamente de secreções de zooxantelas, pois se observava in situ que muitas espécies coletavam e ingeriam zooplâncton.

Vários estudos sobre a abundância do zooplâncton têm mostrado que a fauna recifal remove de 20 a $80 \%$ do holoplâncton da água que passa sobre as cristas e poças (TRANTER; GEORGE, 1972; GLYNN, 1973; JOHANNES; GERBER, 1974; EREZ, 1990). Além disso, a conectividade biológica entre ecossistemas adjacentes, como manguezais e prados de fanerógamas é também de grande relevância em estudos sobre o plâncton recifal, uma vez que muitos organismos realizam migrações ontogenéticas de um habitat a outro durante sua vida. Migrações entre habitats por adultos que têm larvas planctônicas têm sido relatado tanto para invertebrados (HIDDINK, 2003) quanto para vertebrados (LAW; DICKMAN, 1998), sendo a maioria dos estudos com peixes (LENORMAND DODSON, MENARD, 2004; MUMBY, 2006). Estratégias de conservação deveriam proteger corredores de conectividade facilitando a migração de espécies.

Deste modo, considerando a importância dos recifes costeiros, foi desenvolvida a presente pesquisa pelo Departamento de Oceanografia da Universidade Federal de Pernambuco (UFPE), em convênio com a Fundação O Boticário e a World Wildlife Funds (WWF), objetivando conhecer a área de Tamandaré como modelo de uma área recifal tropical costeira do Atlântico sudoeste.

Sobre o zooplâncton desta área os trabalhos publicados são poucos, destacando-se os de Santana-Barreto (1986), Porto-Neto et al. (2000), Nascimento-Vieira (2000) e Silva (2002).

A presente pesquisa objetivou estudar a biodiversidade e variações espaciais e sazonais do mesozooplâncton próximo aos recifes da baía de Tamandaré, com base no pressuposto que estas áreas apresentam alta biodiversidade e abundância quantitativa e tem forte conectividade com os manguezais adjacentes.

\section{CARACTERIZAÇÃO DA ÁREA}

Tamandaré é uma pequena cidade situada a $110 \mathrm{~km}$ ao sul de Recife, Pernambuco, Brasil (08 $45^{\prime} 36^{\prime \prime}$ a $08^{\circ} 47^{\prime} 20^{\prime \prime} \mathrm{S}$ e $35^{\circ} 03^{\prime} 45^{\prime \prime}$ a $35^{\circ} 06^{\prime} 45^{\prime \prime} \mathrm{W}$ ) (Fig. 1). Tem aproximadamente $9 \mathrm{~km}$ de costa dividida em 3 áreas: Praia dos Carneiros, Praia de Campas e Baía de Tamandaré (MAIDA; FERREIRA, 1995). Para esta área foi implantada uma "Área de Proteção Ambiental Costa dos Corais - APA Costa dos Corais" (Decreto Federal de 27 de outubro de 1997), estando este complexo recifal inserido na primeira APA criada para recifes costeiros no Brasil. 
A baía de Tamandaré é uma reentrância na costa formada em decorrência de estrutura de falhamento, típico de litorais do Atlântico (REBOUÇAS, 1966). Limita-se ao norte com o "Pontal do Lira"; ao sul com a Ponta do Mamucabinha, a leste com a linha de recifes e a oeste com a linha de praia. Apresenta-se de forma semi-circular com concavidade voltada para leste e com simetria quase perfeita, levando-se em consideração a linha de contorno entre seus limites norte e sul (REBOUÇAS, 1966). Possui $4 \mathrm{~km}^{2}$ e profundidade em torno de $7 \mathrm{~m}$, com queda suave até a isóbata de $10 \mathrm{~m}$ na saída da barra. O litoral em frente à baía é formado por uma praia estreita com declive acentuado (MABESOONE; COUTINHO, 1970). Próximo ao Pontal do Lira a praia apresenta-se mais larga, com declive suave e profundidade média de $3 \mathrm{~m}$. Na ponta do Mamucabinha o declive também é suave com uma profundidade média é de $0,5 \mathrm{~m}$ ficando parte desta área exposta durante a maré baixa.

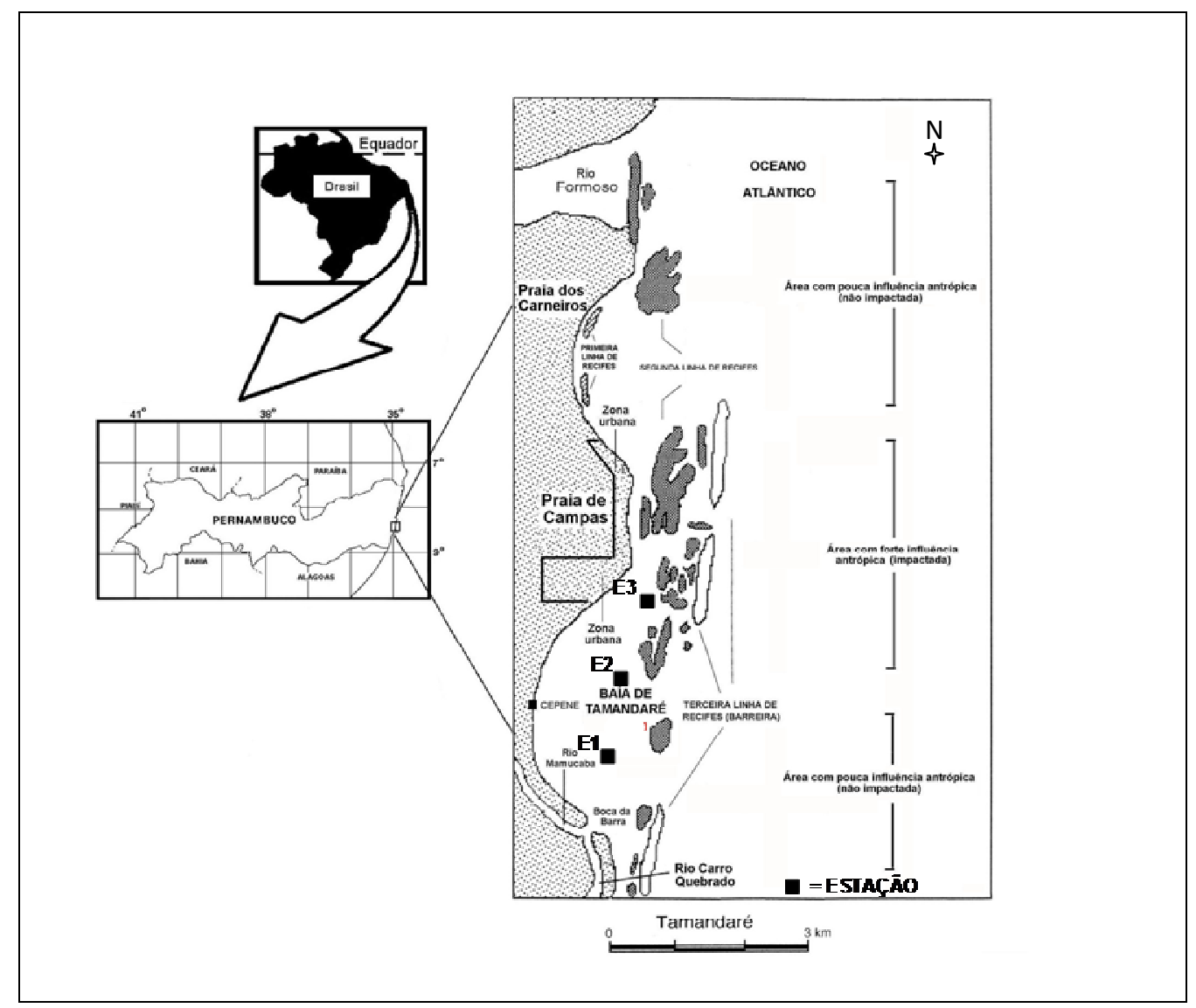

Figura 1 - Localização da área de estudo com as estações na área recifal de Tamandaré, Pernambuco (Brasil) - Atlântico Sudoeste Tropical.

Um dos aspectos importantes é a faixa recifal, que corre paralela a praia e forma construções semelhantes a recifes de franja com uma lagoa relativamente profunda (até $7 \mathrm{~m}$ ). Quatro espécies de corais são encontradas: Mussimilia hispida (Verrill) 1868, Mussimilia hartii (Verrill) 1868 (Família Mussidae), Favia gravida Verrill, 1868 (Família Faviidae) e Siderastrea stellata Verrill, 1868 (Família Siderastreidae), além do hidrocoral Millepora braziliensis (Verrill) 1868 (Família Milleporidae) (MAIDA; FERREIRA, 1995).

Existem três grupos de formações recifais, dispostos quase em linhas paralelas à costa, as quais estão detalhadas em Ferreira, Maida; Souza (1995). A baía de Tamandaré sofre ainda a influencia dos estuários dos rios Ilhetas e Mamucabinha ricos em manguezais.

O clima segundo köepen é do tipo $\mathrm{As}^{\prime}$ (quente e úmido), com precipitação pluviométrica média anual de aproximadamente $2000 \mathrm{~mm}$, sendo as maiores concentrações nos meses de abril a agosto (período chuvoso). A temperatura média 
NASCIMENTO-VIEIRA et al. Mesozooplâncton de área recifal do Atlântico Sudoeste Tropical

anual do ar é geralmente superior a $24^{\circ} \mathrm{C}$, e os ventos dominantes são de SE e NE (NIMER, 1979).

\section{MATERIAL E MÉTODOS}

$\mathrm{Na}$ área estudada foram demarcadas três estações de coletas próximas a área recifal (Fig. 1). A Estação 1 está localizada nas coordenadas geográficas $08^{\circ} 45^{\prime} 48^{\prime \prime} \mathrm{S}$ e $35^{\circ} 05^{\prime} 50^{\prime \prime}$ W. A Estação 2, localizada na região central da baía, nas coordenadas geográficas $08^{\circ} 46^{\prime} 00^{\prime \prime S}$ e $35^{\circ} 05^{\prime} 00^{\prime \prime}$ W. E a Estação 3, localizada no extremo sul da baía, situada a $08^{\circ} 46^{\prime} 30^{\prime \prime} \mathrm{S}$ e $35^{\circ} 06^{\prime} 00^{\prime \prime} \mathrm{W}$.

Os dados pluviométricos foram obtidos do Instituto Nacional de Meteorologia (INMET). As seguintes variáveis abióticas foram também aferidas: temperatura da água superficial $\left({ }^{\circ} \mathrm{C}\right)$, obtida pela leitura direta de termômetro de mercúrio digital; e, salinidade determinada pelo método Mohr-Knudsen, descrito por Strickland; Parsons (1965). As amostras foram coletadas em marés de sizígia, nos regimes de opreamares e baixa-mares, no período de julho/1989 a maio/1990, com intervalo de dois meses entre uma coleta e outra. Esse material encontra-se depositado no Departamento de Oceanografia da UFPE e fornece dados pretéritos da área, antes da implantação da APA Costa dos Corais.

O material coletado foi obtido através de arrastos horizontais à superfície, com duração de 10 minutos cada coleta, nas três estações de coleta com embarcação com motor de popa. As amostras foram obtidas através de rede de plâncton com malha de $300 \mu \mathrm{m}$ de abertura, com um fluxômetro acoplado à boca da rede. Após cada coleta o material foi fixado com formol neutro a 4\%, de acordo com Newell; Newell (1963). Em laboratório, foi obtido o peso úmido de cada amostra de acordo com Omori; Ikeda (1984). Para a análise quali-quantitativa dos organismos mesozooplanctônicos cada amostra foi diluída para um volume de $500 \mathrm{ml}$, homogeneizada e retirada uma subamostra de $4 \mathrm{ml}$ com pipeta tipo Stempel. Cada subamostra foi colocada em placa de Bolgorov e levada ao esteriomicrosscópio Zeiss para triagem, identificação e contagem dos organismos. Foram analisadas um total de três sub-amostras por amostra. Para identificação de algumas espécies foram confeccionadas lâminas, observadas em microscópio composto Zeiss.

No estudo taxonômico e ecológico foram consultadas, dentre outras, as seguintes bibliografias: Tregouboff; Rose (1957), Björmberg (1981) e Boltovskoy (1999). Os dados obtidos foram submetidos a cálculos de abundância relativa (\%), freqüência de ocorrência (\%), biomassa $\left(\mathrm{mg} \cdot \mathrm{m}^{-3}\right)$ e densidade $\left(\mathrm{org} \cdot \mathrm{m}^{-3}\right)$. O índice de diversidade específica baseou-se nos Copepoda sendo utilizado o índice de Shannon (1948) e a eqüitabilidade de Pielou (1969).

\section{RESULTADOS}

A precipitação pluviométrica no período de estudo variou de um mínimo de 24,4 $\mathrm{mm}$ em março de 1990 a um máximo de $464,2 \mathrm{~mm}$ em julho de 1989 . A média mensal da pluviometria no período de 1970 a 1990 variou de 93,1 mm em setembro a um máximo de 396,3 em julho (Fig. 2).

A temperatura variou de $25,1^{\circ} \mathrm{C}$ (Estação 1, baixa-mar, julho/89) a $30,6^{\circ} \mathrm{C}$ (Estação 3, preamar, março/90) (Fig. 3). A salinidade variou de 21,2 (Estação 1, preamar, julho/89) a 34,79 (Estação 1, baixa-mar, maio/90) (Fig. 3). 


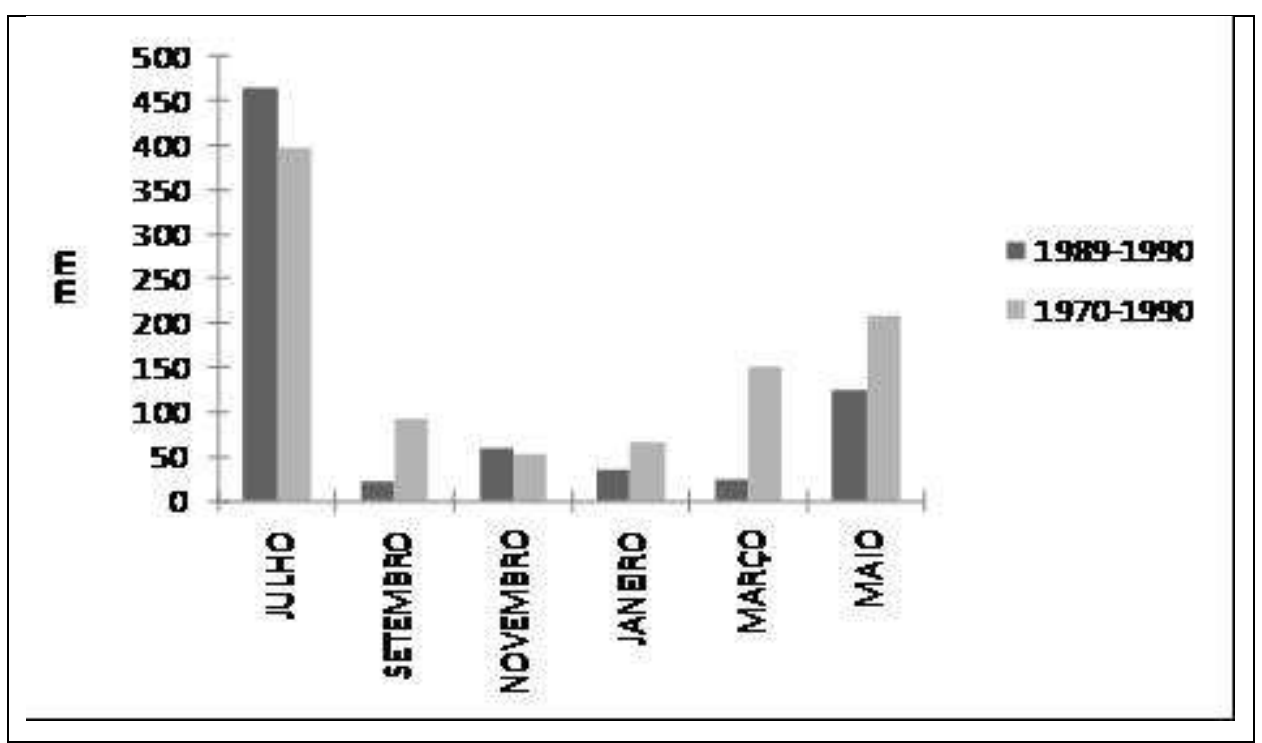

Figura 2 - Precipitação pluviométrica na área de Tamandaré (Pernambuco, Brasil).

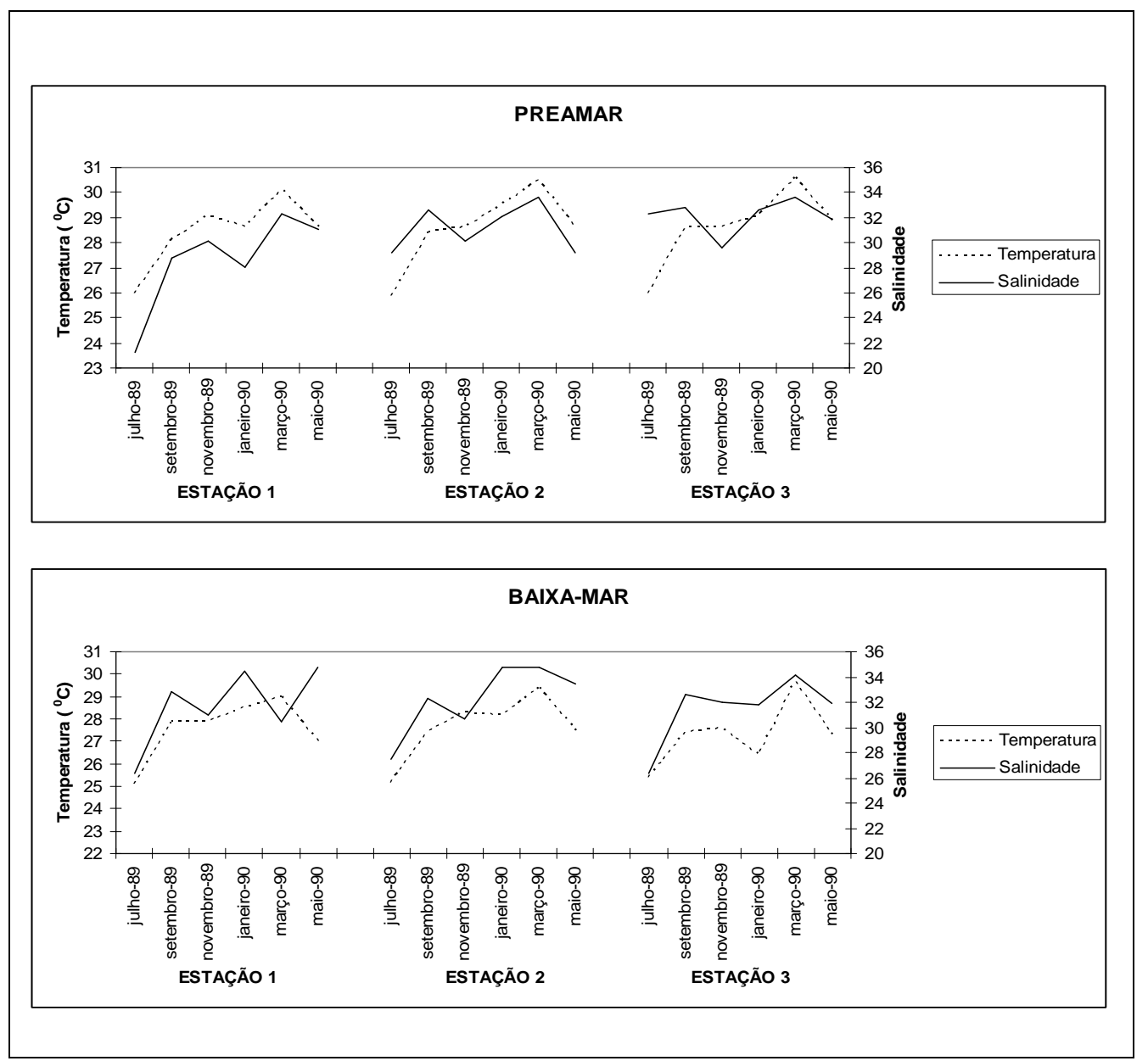

Figura 3 - Temperatura e salinidade nas três estações de coleta na baía de Tamandaré, Pernambuco (Brasil), no período de julho/89 a maio/90.

$\mathrm{Na}$ composição mesozooplanctônica foram identificados 47 taxa considerando a menor unidade taxonômica possível de identificar para cada filo (Tab. I). O holoplâncton predominou sobre os demais membros da comunidade mesozooplanctônica, constituindo $61 \%$ da comunidade total. Dentre os grupos taxonômicos registrados na Estação 1 
NASCIMENTO-VIEIRA et al. Mesozooplâncton de área recifal do Atlântico Sudoeste Tropical

Crustacea (Copepoda) predominou em todos os meses e marés, exceto na preamar de março/90 quando predominaram os Larvacea (Oikopleura dioica Fol, 1872 - Família Oikopleuridae) e as larvas de Polychaeta (incluídas em outros organismos), com cerca de $30 \%$.

Tabela I - Composição da comunidade mesozooplanctônica nas três estações de coleta na baía de Tamandaré, Pernambuco (Brasil), no período de julho/89 a maio/90.

\begin{tabular}{|c|c|}
\hline $\begin{array}{l}\text { Cirripedia } \\
\text { Balanus sp. (náuplio e cípris) } \\
\text { Copepoda } \\
\text { Undinula vulgaris (Dana, 1849) } \\
\text { Parvocalanus crassirostris (Dahl, 1894) } \\
\text { Subeucalanus pileatus (Giesbrecht, 1888) } \\
\text { Centropages velificatus (Oliveira, 1947) } \\
\text { Temora stylifera (Dana, 1849) } \\
\text { Temora turbinata (Dana, 1849) } \\
\text { Labidocera fluviatilis ( Dahl, 1894) } \\
\text { Pontellopsis brevis (Giesbrecht, 1889) } \\
\text { Calanopia americana F. Dahl, 1894 } \\
\text { Acartia (Odontacartia) lilljeborgi Giesbrecht, } \\
\text { 1889 } \\
\text { Oithona oswaldocruzi Oliveira, 1945 } \\
\text { Oithona hebes Giesbrecht, 1891 } \\
\text { Macrosetella gracilis (Dana, 1847) } \\
\text { Euterpina acutifrons (Dana, 1847) } \\
\text { Oncaea media Giesbrecht, 1891 } \\
\text { Oncaea venusta (Philippi, 1843) } \\
\text { Copilia mirabilis Dana, 1849 } \\
\text { Corycaeus (Onychocorycaeus) giesbrechti F. } \\
\text { Dahl, 1894 }\end{array}$ & $\begin{array}{l}\text { Ostracoda } \\
\text { Conchoecia sp. } \\
\text { Mysidacea } \\
\text { Amphipoda } \\
\text { Isopoda } \\
\text { Epicaridea } \\
\text { Cumacea } \\
\text { Eucarida } \\
\text { Euphausiacea } \\
\text { Decapoda } \\
\text { Lucifer faxoni Borradaile,1915 } \\
\text { Lucifer typus H. Milne Edwards, } \\
\text { 1837 } \\
\text { Thalassinidea Latreille, 1831 } \\
\text { Callichirus sp. } \\
\text { Palinuridae } \\
\text { Panulirus sp. (larva) } \\
\text { Porcellanidae (zoea) } \\
\text { Brachyura (zoea e megalopa) } \\
\text { Bryozoa } \\
\text { Membranipora sp. (larva } \\
\text { cifonáutica) } \\
\text { Echinodermata (larva pluteus, } \\
\text { ophiopluteus) } \\
\text { Chaetognatha } \\
\text { Sagitta friderici (Ritter - Zahóny, } \\
\text { 1911) } \\
\text { Chordata } \\
\text { Phlebobranchia } \\
\text { Ascidia sp. (larva) } \\
\text { Oikopleuridae } \\
\text { Oikopleura dioica Fol, 1872 } \\
\text { Oikopleura longicauda (Vogt, } \\
\text { 1854) } \\
\text { Vertebrata } \\
\text { Teleostei (ovos e larvas) }\end{array}$ \\
\hline
\end{tabular}

Na Estação 2, o predomínio foi também de Crustacea (Copepoda), exceto na baixamar de setembro/89, quando Teleostei (ovos) se destacaram compondo o grupo "outros organismos"; e, na preamar de julho/89 quando Sagitta friderici Ritter-Zahóny, 1911 (Família Sagittidae) foi a espécie dominante, estando também inserida dentro de "outros organismos" (Fig. 4). Para a estação 3, Crustacea (Copepoda) foram os organismos que mais se destacaram, exceto nas baixa-mares de setembro/89 (dominou Teleostei - ovos) e maio/90 (quando predominou Brachyura-zoea, e outras Decapoda-larvas) e na preamar de março/90, quando se destacaram Oikopleura dioica e os zoea de Brachyura (Fig. 4). 


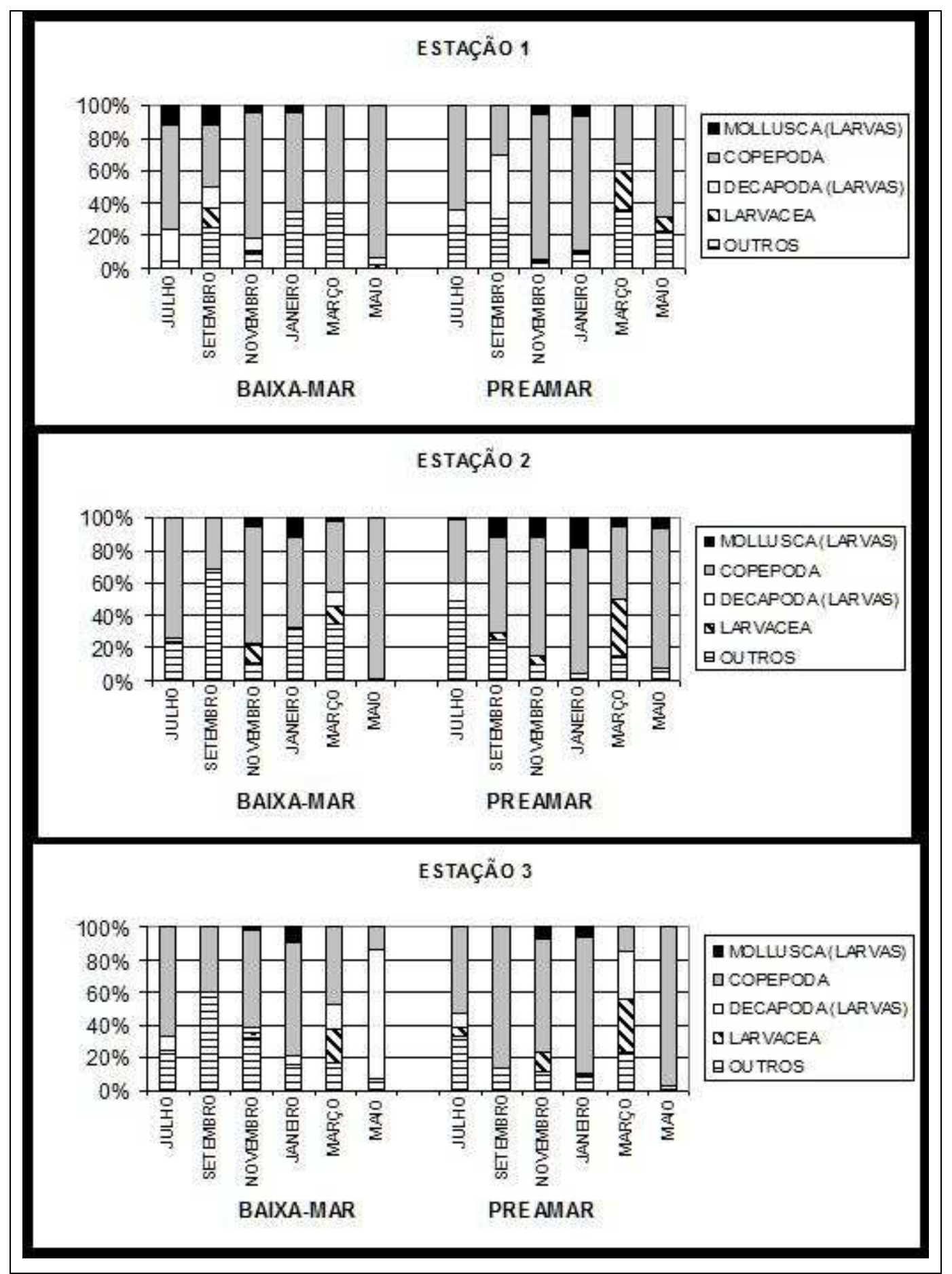

Figura 4 - Abundância relativa do mesozooplâncton coletado nas três estações de coleta na baía de Tamandaré, Pernambuco (Brasil), no período de julho/89 a maio/90.

No tocante à freqüência de ocorrência foram considerados muito freqüentes Acartia lilljeborgi (100\%) e Parvocalanus crassirostris (80,56\%), constituindo $5,5 \%$ da comunidade mesozooplanctônica. Dentre os freqüentes ocorreram ovos de Teleostei $(63,89 \%)$, náuplios e cípris de Cirripedia (52,78\%), Calonopia americana F. Dahl, 1894 (Família Pontellidae), Corycaeus (Onychocorycaeus) giesbrechti F. Dahl, 1894 (Família Corycaeidae) e zoea de Brachyura, com $50 \%$ e Sagitta friderici $(44,4 \%)$. Os demais táxons variaram de pouco freqüentes a raros, sendo que destes $61 \%$ ocorreram em menos de $30 \%$ das amostras. 
NASCIMENTO-VIEIRA et al. Mesozooplâncton de área recifal do Atlântico Sudoeste Tropical

A biomassa sestônica variou de $9,24 \mathrm{mg} \cdot \mathrm{m}^{-3}$ a 73,94 $\mathrm{mg} \cdot \mathrm{m}^{-3}$ (Fig. 5). A média geral foi $24,4 \pm 5,9 \mathrm{mg} \cdot \mathrm{m}^{-3}$. Na Estação 1 a preamar apresentou biomassa superior a registrada durante a baixa-mar; na Estação 2 a preamar apresentou maiores biomassas em julho e setembro/89 e as baixa-mares em março e maio/90, os demais meses foram semelhantes. Para a Estação 3 as baixa-mares apresentaram biomassas maiores que as preamares, mostrando comportamento inverso ao da Estação 1.

Em termos de densidade, foi observado um ciclo sazonal com maiores valores nos meses de verão nas três estações, exceto o pico registrado para a Estação $1 \mathrm{em}$ maio/90. A Estação 1 apresentou maior densidade durante a preamar, enquanto que a Estação 3 apresentou maiores densidades durante a baixa-mar. A densidade mínima geral foi de $24 \mathrm{org} . \mathrm{m}^{-3}$ (Estação 3, preamar de setembro/89) e máxima de $992 \mathrm{org} . \mathrm{m}^{-3}$ (Estação 1, preamar de novembro/89) (Fig. 6).

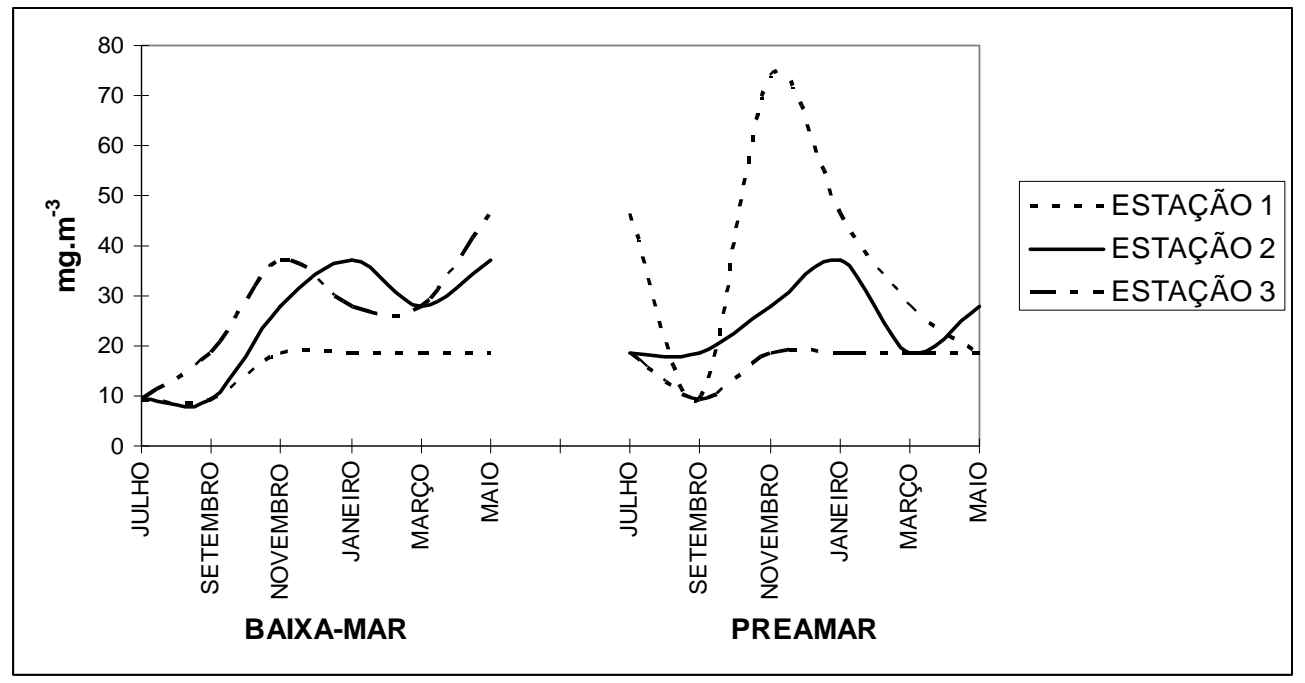

Figura 5 - Biomassa do mesozooplâncton coletado nas três estações de coleta na baía de Tamandaré, Pernambuco (Brasil), no período de julho /89 a maio/90.

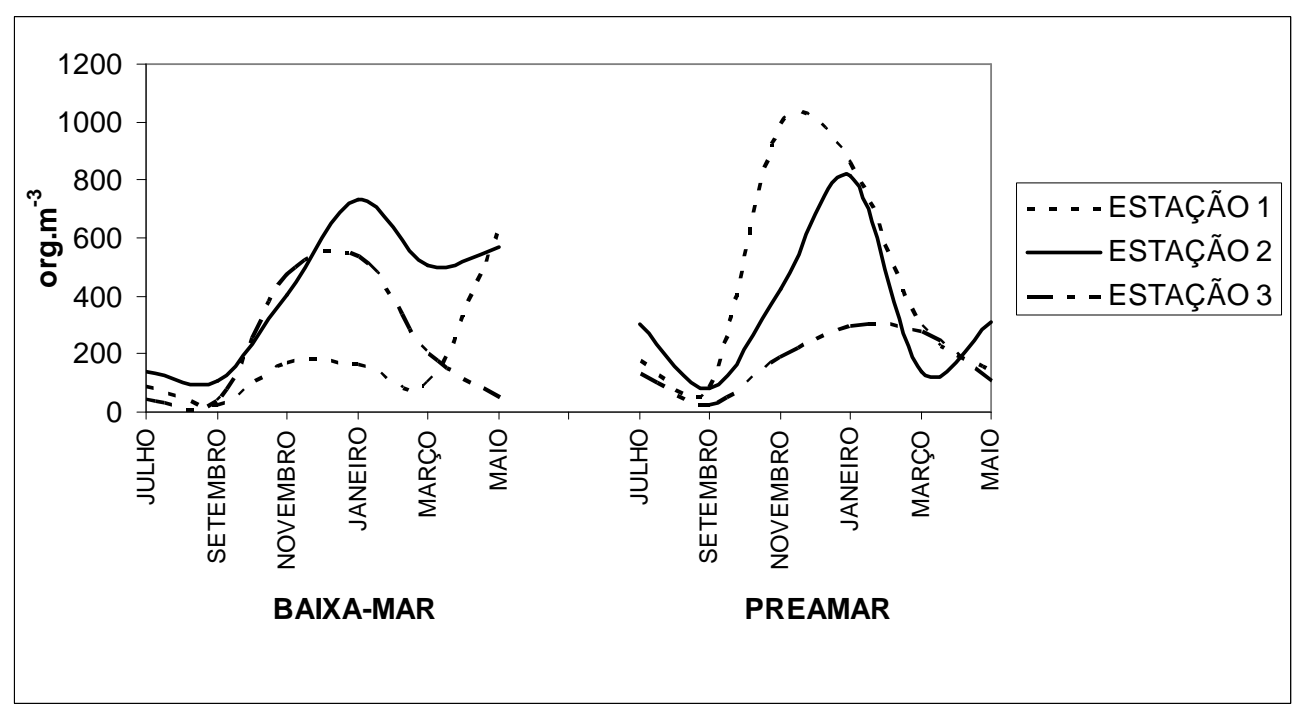

Figura 6 - Densidade do mesozooplâncton coletado nas três estações de coleta na baía de Tamandaré, Pernambuco (Brasil), no período de julho /89 a maio/90.

Em termos de diversidade específica média durante a baixa-mar foi de 2,47 $\pm 0,71$ bits.ind ${ }^{-1}$, sendo um pouco menor que a média geral da preamar $\left(2,56 \pm 0,37\right.$ bits.ind $\left.^{-1}\right)$. $\mathrm{Na}$ Estação 1 o mínimo foi de 1,96 bits.ind $^{-1}$ na baixa-mar de maio/90 e o máximo foi 3,47 bits.ind $^{-1}$ na baixa-mar de novembro/89 (Fig. 7). Para a Estação 2 os valores variaram de 1,74 bits.ind $^{-1}$ na baixa-mar de setembro/89 a 2,98 bits.ind ${ }^{-1}$ na preamar de 
março/90 (Fig. 7). Para a Estação 3 os valores variaram de 0,92 bits.ind $^{-1}$ (maio/90) a 3,92 bits.ind ${ }^{-1}$ (novembro/89) ambos na baixa-mar (Fig. 7). Este baixo valor decorreu do pequeno número de espécies registradas. De uma forma geral, as baixa-mares de novembro/89 apresentaram as maiores diversidades e as preamares de maio/90 os menores.

Quanto à eqüitabilidade (Fig. 8), os valores foram sempre maiores que 0,5 indicando uma boa distribuição entre os organismos mesozooplanctônicos ao longo da coluna d'água. O mínimo para a área foi 0,56 na baixa-mar de maio/90 estação 2 e máximo de 0,96 na baixa-mar de setembro/89, na estação 3.

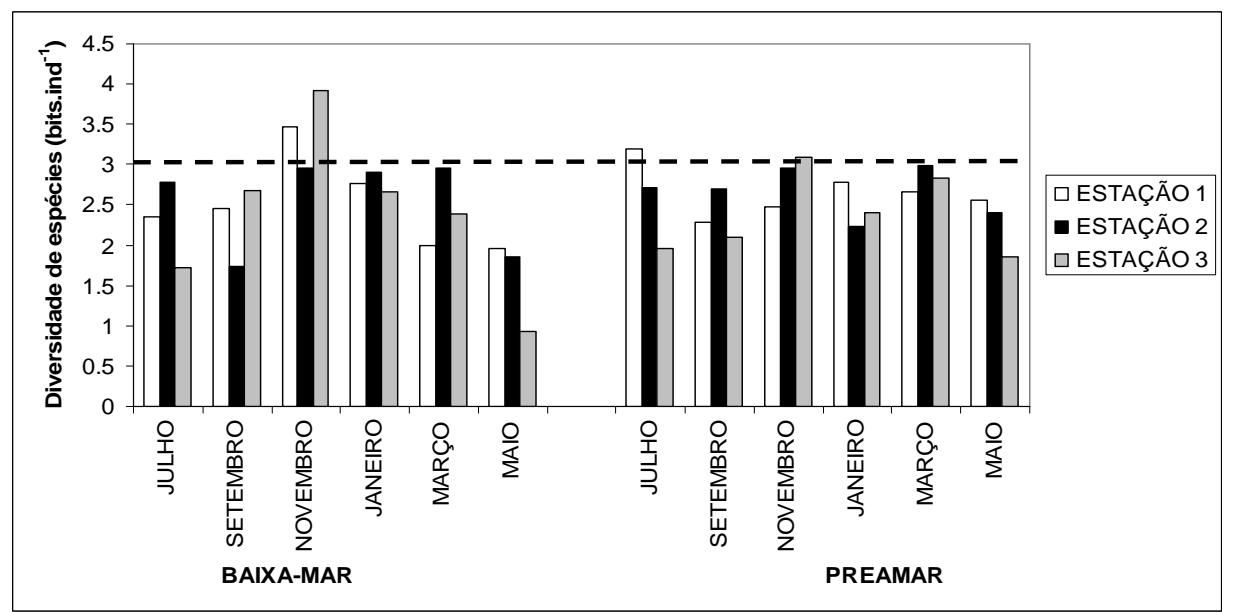

Figura 7 - Diversidade de espécies de Crustacea (Copepoda) nas três estações de coleta na baía de Tamandaré, Pernambuco (Brasil), no período de julho /1989 a maio/1990.

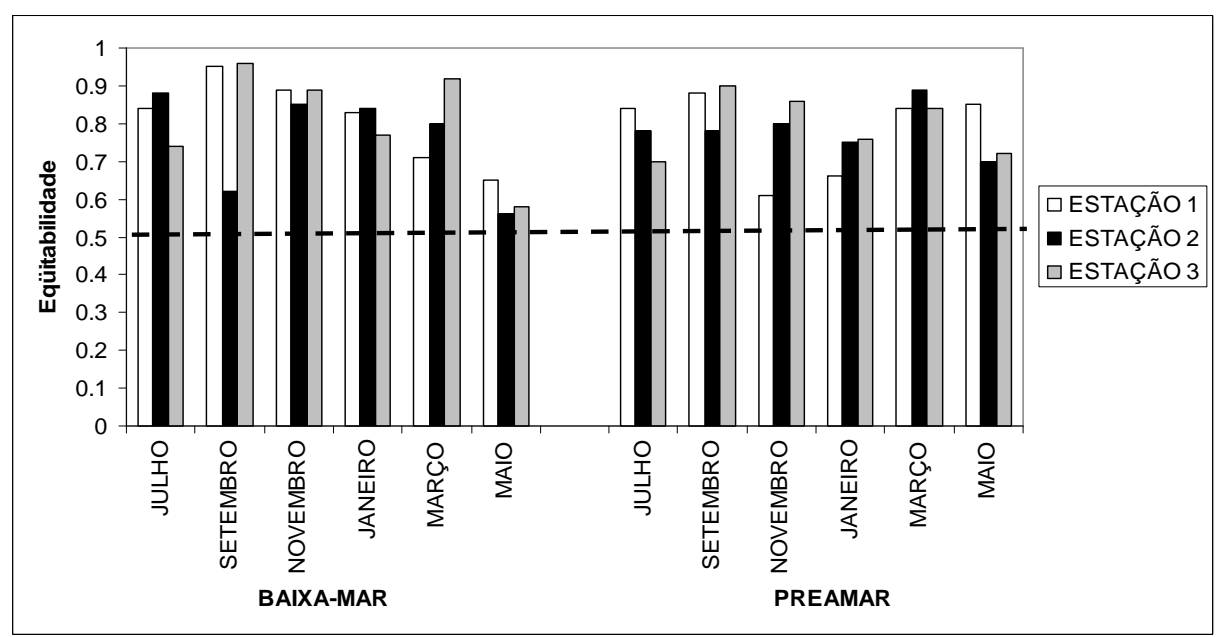

Figura 8 - Eqüitabilidade de Crustacea (Copepoda) nas três estações de coleta na baía de Tamandaré, Pernambuco (Brasil), no período de julho /89 a maio/90.

\section{DISCUSSÃO}

A área recifal de Tamandaré caracteriza-se pela presença de uma massa d'água costeira com forte influência da pluma estuarina (MACEDO, 2009), com amplitudes de até 15 de salinidade no período chuvoso, contribuindo, possivelmente, para a redução da biodiversidade zooplanctônica. Foram registrados 47 taxa, sendo os Crustacea (Copepoda) representados por 18 espécies. O predomínio na área é de espécies marinhas eurialinas, encontradas em grandes quantidades nos estuários adjacentes. Estudos realizados no ecossistema recifal de Maracajaú (Rio Grande do Norte) com mesma malha de rede e desenho amostral semelhante mostrou um zooplâncton com diversidade um pouco menor com 40 taxa, destacando-se Crustacea (Copepoda) com 15 espécies (MELO et al., 2002). 
NASCIMENTO-VIEIRA et al. Mesozooplâncton de área recifal do Atlântico Sudoeste Tropical

Dentre as espécies marinhas eurialinas holoplanctônicas destacaram-se em Tamandaré os Crustacea (Copepoda) Acartia lilljeborgi e Parvocalanus crassirostris, principalmente nas baixa-mares, seguidos pelo Larvacea Oikopleura dioica e pelo Chaetognatha Sagitta friderici. Dentre osmeroplanctônicos destacaram-se as zoeas de Brachyura, além das larvas de Balanus, véliger de Bivalvia, larvas de Polychaeta e larvas de Teleostei. Estes formam um grupo comumente encontrado nos estuários brasileiros (NEUMANN-LEITÃO, 1995).

Parvocalanus crassirostris apresenta grande tolerância à salinidade, estando limitado às águas costeiras de regiões tropicais e subtropicais (BJÖRNBERG, 1981), sendo esta espécie a mais comumente citada em águas costeiras e estuarinas do Brasil, com exceção da lagoa dos Patos (MONTÚ, 1980).

Acartia lilljeborgi domina com frequencia águas tropicais costeiras e estuarinas com salinidade mais elevada (BJÖRNBERG, 1981), tendo sido registrada para diversos estuários e áreas costeiras de Pernambuco (NASCIMENTO, 1981; PARANAGUÁ, 1986; NEUMANN-LEITÃO, 1995; ESKINAZI-SANT'ANNA; TUNDISI, 1996; NEUMANN-LEITÃO et al., 1999; SILVA et al., 2003; SILVA et al., 2004).

A presença de Brachyura nas fases zoea e megalopa decorre possivelmente da presença das fêmeas adultas nos estuários/manguezais adjacentes e que são transportadas para a região recifal. Em estudos nos manguezais do Canal de Santa Cruz, Schwamborn (1997) observou que zoeas de Brachyura eram exportadas para a área recifal costeira, sendo encontradas em grande abundância até 10-20 km da costa. Um estudo da distribuição fotoperiódica e sazonal das zoeas de Brachyura em Itamaracá (SILVA-FALCÃO, SEVERI, ROCHA, 2007), ao norte da área estudada, evidenciou que as larvas de Ocypodidae e Grapsidae indicaram um movimento de dispersão para áreas costeiras, enquanto que os Pinnotheridae indicaram uma provável retenção nas águas do estuário. O movimento de dispersão em direção à área costeira pode estar relacionado à salinidade, pois segundo González-Gordillo; Rodriguez (2003), a ocorrência de baixa salinidade parece inibir ou dificultar o desenvolvimento larval de muitas espécies de Decapoda. Experimentos de cultivo em laboratório evidenciaram que a salinidade igual ou próxima à do mar é ótima para o completo desenvolvimento larval de vários Brachyura (FRANSOZO, 1987; ANGER et al., 1990; LUPPI, SPIVAK; BAS, 2003).

A pequena quantidade de táxons dominantes resultou numa diversidade específica com valores médios. Esperava-se uma maior diversidade uma vez que áreas recifais são tidas como áreas de grande biodiversidade. Porto Neto et al. (2000) também encontraram diversidade média para o zooplâncton coletado com auxílio de uma bomba em várias profundidades na baía de Tamandaré.

Em termos quantitativos foi observado para Tamandaré um padrão sazonal com maiores densidades no período seco, já no sistema recifal de Maracajaú no Rio Grande do Norte as maiores densidades foram registradas no período chuvoso, alcançando, porém, valores menores do que em Tamandaré (MELO et al., 2002).

Pode-se sugerir que a diversidade do zooplâncton, em Tamandaré (Pernambuco, Brasil) foi influenciada pelas correntes de marés, precipitação pluviométrica e drenagem continental. Nestas condições, Paula et al. (1998) ressaltaram a importância da hora da coleta e do local da estação em relação à drenagem continental, e afirmaram que ambientes costeiros "tipo mosaico" determinam a diversidade do plâncton.

É interessante observar, que as maiores biomassas e densidades zooplanctônicas em Tamandaré foram registradas nos períodos secos e nos regimes de preamares. Os resultados sugerem a existência de um período favorável ao mesozooplâncton recifal que vai de novembro a fevereiro, quando a temperatura é um pouco mais elevada e a influência da drenagem continental é menor. Desta forma, a precipitação pluviométrica parece ser um dos principais fatores na estruturação da biodiversidade do mesozooplâncton na baía de Tamandaré (Pernambuco, Brasil). 


\section{AGRADECIMENTOS}

Os autores agradecem ao Dr. Reinaldo Tenório pela coleta das amostras e ao Dr. José Zanon de Oliveira Passavante, Chefe do Laboratório de Plâncton pela cessão das amostras do acervo do Departamento de Oceanografia da UFPE. À Dra. Kátia Muniz pela análise das amostras de salinidade.

\section{REFERÊNCIAS}

ANGER, K.; MONTÚ, M.; BAKKER, C.; FERNANDES, L. L. Larval development of Uca thayeri Rathbun, 1900 (Decapoda:Ocypodidae) reared in the laboratory. Meeresforsch, v. 32, n.1, p.:276-294, 1990.

BJÖRNBERG, T. S. Copepoda., p. 587-679. In: D. Boltovskoy (Ed.). Atlas del zooplancton del Atlántico sudoccidental y métodos de trabajo con el zooplancton marino. Mar del Plata, INIDEP, 1981, 936p.

BOLTOVSKOY, D. South Atlantic Zooplankton. Leiden, Backhuys Publishers, 1999, $1627 p$.

EREZ, J. On the importance of food sources in coral-reef ecosystems, p. 411-418. In: Z. Dubinsky (Ed.), Ecosystems of the World, Coral Reefs, Volume 25, Amsterdam, Elsevier, 1990. 550p.

ESKINAZI-SANT'ANNA, E. M.; TUNDISI, J. G. Zooplâncton do estuário do Pina (RecifePernambuco-Brasil): composição e distribuição temporal. Revista Brasileira de Oceanografia, São Paulo, v. 44, n.1, p. 23-33, 1996.

FERREIRA, B. P.; MAIDA, M.; SOUZA, A. E. T. Levantamento inicial das comunidades de peixes recifais da região de Tamandaré - PE. Boletim Técnico-Científico do CEPENE, Tamandaré, v.3, n.1, p. 211-230, 1995.

FRANSOZO, A. Desenvolvimento larval de Eriphia gonagra (Fabricius, 1781) (Decapoda, Xanthidae), em laboratório. Revista Brasileira de Zoologia, v.4, n.3, p.165-179, 1987.

GLYNN, P. W. Ecology of Caribbean coral reef; plankton community with evidence for depletion. Marine Biology, Berlin, v.22, p. 1-23, 1973.

GONZÁLEZ-GORDILLO, J. L.; RODRIGUEZ, A. Comparative seasonal and spatial distribution of decapod larvae assemblages in three coastal zones off the south-western Iberian Peninsula. Acta Oecologica, v.24, n.1, p.219-233, 2003.

GROSS, M. G.; GROSS, E. Oceanography: A View of Earth. New Jersey, Pretice-Hall Inc., 1996. 472p.

HIDDINK, J.G. Modelling the adaptive value of intertidal migration and nursery use in the bivalve Macoma balthica. Marine Ecology-Progress Series, v.252, p.173-185, 2003.

JOHANNES, R. E. Pollution and degradation of coral reef communities, p. 13-50. In: E. J. Ferguson Wood; R. E. Johannes (Ed.). Tropical marine pollution. Amsterdam, Elsevier Scientific Publishing, 1975, X+192p.

JOHANNES, R. E.; GERBER, R. P. Import and export of plankton by Eniwetok reef community. Proceedings of the Second International Coral Reef Symposium, Brisbane, v.1, p. 97-104, 1974.

LAW, B.S.; DICKMAN, C.R.; The use of habitat mosaics by terrestrial vertebrate fauna: Implications for conservation and management. Biodiversity and Conservation, v.7, p.323-333, 1998.

LENORMAND, S.; DODSON, J.J.; MENARD, A. Seasonal and ontogenetic patterns in the migration of anadromous brook charr (Salvelinus fontinalis). Canadian Journal of Fisheries and Aquatic Sciences, v.61, p.54-67, 2004.

LUPPI, T. A.; SPIVAK, E. D.; BAS, C. C. The effects of temperature and salinity on larval development of Armases rubripes Rathbun, 1897 (Brachyura, Grapsoidea, Sesarmidae), 
NASCIMENTO-VIEIRA et al. Mesozooplâncton de área recifal do Atlântico Sudoeste Tropical

and the southern limit of its geographical distribution. Estuarine, Coastal and Shelf Science, v.58, n.3, p.575-585, 2003.

MABESOONE, J. M.; COUTINHO, P. Littoral and shallow marine geology of northeastern, Brasil. Trabalhos Oceanográficos da Universidade Federal de Pernambuco, Recife, v.12, p.1-214, 1970.

MACEDO, E. C. Um ensaio sobre o sedimento e suas implicações ecológicas nos recifes costeiros da baia de Tamandaré/PE. http://biblioteca.universia.net/autor/ Eduardo\%20Cavalcante\%20de\%20Macêdo.html (PDF) - 18-oct-2009

MAIDA, M.; FERREIRA, B. P. Estudo preliminar sobre o assentamento de corais em um recife na baía de Tamandaré - PE. Boletim Técnico Científico do CEPENE, Tamandaré, v.3, n.1, p.23-36,1995.

MELO, N.F.A.C; NEUMANN-LEITÃO, S.; SILVA, T. A.; SCHWAMBORN, R. ; GUSMÃO, L. M. O. Zooplankton from the Maracajaú reefs, Northeastern Brazil. Tropical Oceanography, v. 30, n. 2, p. 133-145, 2002.

MONTÚ, M. Zooplâncton do estuário da Lagoa dos Patos. I. Estrutura e variações temporais e espaciais da comunidade. Atlântica, Rio Grande, v.4, p.53-72, 1980.

MUMBY, P. J. Connectivity of reef fish between mangroves and coral reefs: Algorithms for the design of marine reserves at seascape scales. Biological Conservation, v. 128, p. 215-222, 2006.

NASCIMENTO, D. A. Estudo Ecológico da região de Itamaracá, Pernambuco - Brasil. XV. Copepoda do estuário do Rio Botafogo. Trabalhos Oceanográficos Universidade Federal de Pernambuco, Recife, v.16, p. 65-88, 1981.

NEUMANN-LEITÃO, S. Resenha Literária Sobre Zooplâncton Estuarino no Brasil. Trabalhos Oceanográficos Universidade Federal de Pernambuco, Recife, v.23, p. 25-53,1995.

NEUMANN-LEITÃO, S.; GUSMÃO, L. M.O.; SILVA, T. A.; NASCIMENTO-VIEIRA, D. A.; SILVA, A. P. Mesozooplankton biomass and diversity in coastal and oceanic waters off North-Eastern Brazil. Archives of Fishery Marine Research, Jena, v.47 n.2-3, p.153$165,1999$.

NEW, T. R. An Introduction to Invertebrate Conservation Biology. Oxford, Oxford Sscience Puublication, 1995. 194p.

NEWELL, G.H.; NEWELL, R.C. Marine plankton: a practical guide. London, Hutchinson Educat, 1963, 221p.

NIMER, E. Pluviometria e recursos hídricos dos Estados de Pernambuco e Paraíba. Rio de Janeiro, IBGE, 1979.117p.

OMORI, M.; IKEDA, T. Methods in marine zooplankton ecology. New York, Wileyinterscience Publication, 1984. 331p.

PARANAGUÁ, M.N. Zooplankton of the Suape area (Pernambuco-Brazil). Trabalhos Oceanográficos Universidade Federal de Pernambuco, Recife, v.19, p.113-124, 1986.

PAULA, J.; PINTO, I.; GUAMBE, S.; MONTEIRO, D.; GOVE, J. GUERREIRO. Seasonal cycle of planktonic communities at Inhaca Island, southern Mozambique. Journal of Plankton Research, Oxford, v. 20, n.11, p. 2165-2178, 1998.

PIELOU, E. C. An introduction to Mathematical Ecology. New York, WileyInterscience, 1969. 286p.

PORTO NETO, F. F.; S. NEUMANN - LEITÃO, D. A. NASCIMENTO VIEIRA, A. P. SILVA, T. A. SILVA; M. C. O. MOURA. Zooplâncton recifal de Tamandaré-PE (Brasil) e a influência dos manguezais na sua biodiversidade e biomassa. In: Mangrove 2000. Sustentabilidade de 
Estuários e Manguezais: Desafios e Perspectivas. Anais... Recife: 2000. Universidade Federal Rural de Pernambuco; ISME, CD-ROM.

REBOUÇAS, A. C. Sedimentos da Baía de Tamandaré - PE. Trabalhos Oceanográficos Universidade Federal de Pernambuco, Recife, v. 7/8, p. 187- 205, 1966.

SANTANA-BARRETO, M. S. Biomassa, densidade e composição do zooplâncton da Baía de Tamandaré. Caderno Ômega, Recife, v.2, p. 55-65, 1986.

SCHWAMBORN, R. Influence of mangroves on community structure and nutrition of macrozooplankton in Northeast Brazil. ZMT Contribution, Bremen, v.4, p. 1- 478, 1997.

SHANNON, C. E. A mathematical theory of communication. Bell System Technical Journal, San Diego, California, v.27, p. 379-423, 1948.

SILVA, T.; NEUMANN-LEITÃO, S.; SCHWAMBORN, R.; GUSMÃO, L. M. O.; NASCIMENTOVIEIRA, D.A. Diel and seasonal changes in the macrozooplankton community of a tropical estuary in Northeastern Brazil. Revista Brasileira de Zoologia, Curitiba, v. 20, p. 439446, 2003.

SILVA, A. P.; NEUMANN-LEITÃO, S.; SCHWAMBORN, R.; GUSMÃO, L. M. O.; SILVA, T. A. Mesozooplankton of an impacted bay in North Eastern Brazil. Brazilian Archives of Biology and Technology, Curitiba, v. 47, p.485-493, 2004.

SILVA-FALCÃO, E.; SEVERI, W.; ROCHA, A. A. F. Dinâmica espacial e temporal de zoeas de Brachyura (Crustacea, Decapoda) no estuário do Rio Jaguaribe, Itamaracá, Pernambuco, Brasil Iheringia, Sér. Zool. Porto Alegre, v.97, n.4, 2007.

SOROKIN, Y. I. Plankton in the reef ecosystems, p. 291-327. In: Z. Dubinsky (Ed). Ecosystems of the World, Coral Reefs, Volume 25, Amsterdam, Elsevier, 1990. 550p.

STRICKLAND, J.D.H.; PARSONS, T.R.A. A manual of seawater analysis. Bulletin of Fisheries Research Board of Canada, Ottawa, v.125, p.1-205, 1965.

TRANTER, D.J.; GEORGE, J. Zooplankton abundance at Kavaratti and Kalpeni Atolls in the Laccadives. Proceedings of the First Symposium on Coral and Coral Reefs, Conchin, v. 1, p. 239-256, 1972.

TREGOUBOFF, G.; ROSE, M. Manuel de planctologie mediterranèenne. Paris, Centre Nacionale de la Recherche Scientifique, 1957. II+584p. 